\title{
Pandemische Grippe (H1N1): Impfobligatorium für das Gesundheitspersonal?
}

Roger Rudolph

Dr. iur., Rechtsanwalt, Autor und Co-Autor verschiedener Kommentare und Abhandlungen zum Zivil- und Arbeitsrecht
Seit Anfang November 2009 hat die Anzahl bestätigter Fälle der Pandemischen Grippe (H1N1) 2009, auch als Schweinegrippe bekannt, stark zugenommen. Spätestens mit dem Bekanntwerden erster Todesfälle in Spitälern wurde der Ruf nach einem Impfobligatorium für die Beschäftigten im Gesundheitswesen laut. Damit einher ging eine intensiv und teils polemisch geführte Kontroverse zwischen Impfbefürwortern und Impfgegnern. Während die Befürworter den Gegnern Unprofessionalität und Gleichgültigkeit gegenüber dem Patientenwohl vorwarfen, sahen die Gegner in der Forderung nach einem Impfobligatorium einen Angriff auf ihre körperliche Integrität und fundamentale verfassungsmässige Rechte.

\section{Impfobligatorium gestützt auf das Epidemiengesetz}

Vergleichsweise klar ist die Rechtslage in Bezug auf das bundesrechtliche Epidemiengesetz, das derzeit zur Revision ansteht. Der heute geltende Art. 23 räumt den Kantonen das Recht ein, ein Impfobligatorium gegen übertragbare Krankheiten auszusprechen, sofern für die Bevölkerung eine erhebliche Gefahr besteht. Eine Rechtsgrundlage zur Einführung einer generellen Impfpflicht ist demnach, entgegen anderslautender Aussagen in der öffentlichen Diskussion, bereits nach geltendem Recht durchaus gegeben. Allerdings ist es so, dass - so weit bekannt - noch von keinem Kanton ernsthaft erwogen wurde, eine solche auf das Epidemiengesetz gestützte Impfpflicht tatsächlich auszusprechen.
[1]. Zum gleichen Schluss kam das St. Galler Verwaltungsgericht in einem Urteil vom 19. Oktober 2006 [2]. In diesem interessanten Entscheid wurde die Weigerung einer Rotkreuzpflegehelferin am Kantonsspital St. Gallen, sich einer vorgeschriebenen HepatitisB-Impfung zu unterziehen, als pflichtwidriges Verhalten gewertet. Die vom Spital deswegen ausgesprochene Kündigung wurde folglich als gerechtfertigt taxiert.

Ein weiterer Ansatz für die Zukunft wäre, dass Impfobligatorien vertraglich zwischen Arbeitgeber und Arbeitnehmer vereinbart werden. Diese durchaus prüfenswerte Möglichkeit würde in erster Linie den privatrechtlichen Arbeitgebern zur Verfügung stehen, d. h. den Privatspitälern und Praxen, da sie dem Obligationenrecht (OR) und damit dem Grundsatz der Vertragsfreiheit unterstehen. Dies im Unterschied zu den öffentlichen Spitälern, die in der Regel in relativ starre Personalrechte eingebunden sind. Es stellt sich allerdings die schwierige und bis heute unbeantwortete Frage, ob eine solche vertraglich vereinbarte Impfpflicht mit dem Verbot übermässiger Selbstbindung von Art. 27 Abs. 2 des Zivilgesetzbuchs vereinbar wäre.

\section{Im Fokus: das Weisungsrecht des Arbeitgebers} Weil von diesen gesetzlichen und vertraglichen Möglichkeiten bis heute kaum je Gebrauch gemacht wurde, muss sich die aktuelle Diskussion auf das sogenannte Weisungsrecht des Arbeitgebers konzentrieren. Dieses Weisungsrecht ist für die privatrechtlichen Arbeitsverhältnisse in Art. 321d Abs. 1 OR ausdrücklich vorgesehen. Danach kann der Arbeitgeber über die Aus-

\section{Das gesetzliche Weisungsrecht des Arbeitgebers kann als rechts- dogmatische Grundlage für ein Impfobligatorium herangezogen werden}

Eine ausführliche Fassung dieses Beitrags findet sich unter www.saez.ch $\rightarrow$ Aktuelle Ausgabe oder $\rightarrow$ Archiv $\rightarrow 2010 \rightarrow 1 / 2$

Korrespondenz:

Dr. iur. Roger Rudolph

Streiff Pellegrini \& von Kaenel

Rechtsanwälte

Bahnhofstrasse 67

CH-8620 Wetzikon

rudolph@streiffpartner.ch

www.streiffpartner.ch
Denkbar wäre auch, dass die Kantone ergänzend zum Epidemiengesetz Impfvorschriften für exponierte Bevölkerungsgruppen wie das Gesundheitspersonal erlassen, z. B. in den kantonalen Personal- oder Gesundheitsgesetzen. Davon haben sie bis heute allerdings noch kaum Gebrauch gemacht. Solche Grundrechtsbeschränkungen wären zulässig, wenn sie auf einer gesetzlichen Grundlage beruhen, von einem öffentlichen Interesse getragen und verhältnismässig sind. Das Bundesgericht hat dies für ein generelles DiphtherieImpfobligatorium für Kinder bereits einmal bejaht führung der Arbeit und das Verhalten des Arbeitnehmers am Arbeitsplatz allgemeine Anordnungen und individuelle Weisungen erlassen. Auch die öffentlichrechtlichen Personalrechte kennen vergleichbare Umschreibungen und Weisungsbefugnisse. Unbestritten ist, dass auch der Schutz der Gesundheit von Arbeitnehmer, Mitarbeitenden und Kunden vom Weisungsbereich erfasst ist. Verschiedene Gesetzesbestimmungen verpflichten den Arbeitgeber sogar, Schutzvorkehrungen zu treffen, so z. B. die gesetzliche Fürsorgepflicht von Art. 328 OR, Art. 6 des Arbeitsgesetzes (ArG) oder 
Art. 82 Abs. 1 des Unfallversicherungsgesetzes (UVG). Man könnte sich fragen, ob sich gestützt auf diese Gesetzesbestimmungen sogar direkt eine Impfpflicht herleiten liesse. Zumindest aber lässt sich sagen, dass das gesetzliche Weisungsrecht des Arbeitgebers als rechtsdogmatische Grundlage für ein Impfobligatorium herangezogen werden kann. Zum gleichen Schluss kam auch das St. Galler Verwaltungsgericht im vorhin erwähnten Urteil aus dem Jahr 2006. Es bejahte die Pflicht zur Vornahme der Hepatitis-B-Impfung, direkt gestützt auf das Weisungsrecht des Spitals und unab-

\section{Wichtig ist nun aber, dass das Weisungsrecht des Arbeitgebers kein unbeschränktes ist. Es findet seine Grenzen insbesondere im Persönlichkeitsrecht} des Arbeitnehmers

hängig davon, ob nun auch noch eine (vertragliche) Zustimmung vorlag oder nicht.

Wichtig ist nun aber, dass das Weisungsrecht des Arbeitgebers kein unbeschränktes ist. Es findet seine Grenzen insbesondere im Persönlichkeitsrecht des Arbeitnehmers (Art. 328 OR). Dabei ist immer eine Interessenabwägung vorzunehmen. Auf Arbeitnehmerseite steht sein Selbstbestimmungsrecht über die eigene körperliche Integrität ganz im Vordergrund. Aufseiten des Arbeitgebers ist in erster Linie sein Interesse an der Aufrechterhaltung eines sicheren Betriebs zu nennen. Dazu gehört auch der Schutz der Mitarbeitenden und Patienten vor vermeidbaren Gesundheitsgefährdungen. Es liegt auf der Hand, dass die Gewichtung der Interessen massgeblich vom konkreten Bedrohungspotential einer Pandemie beeinflusst wird. Je mehr Fälle in einem Spital auftreten, je schwerwie-

Im Sinne der Verhältnismässigkeit ist zu prüfen, welche Schutzmassnahmen Vorrang haben. gender die Symptome sind und je höher die Ansteckungsgefahr ist, um so eher wird das Arbeitgeberinteresse an einer Impfpflicht überwiegen und vice versa. Weiter ist das Verhältnismässigkeitsgebot zu beachten. Daraus folgt, dass mildere Massnahmen, z. B. das Tragen von Masken, den Vorrang haben müssen, solange diese einen zuverlässigen Schutz gewährleisten können. Schliesslich haben sich einseitig vom Arbeitgeber angeordnete Massnahmen wie eine Impfoder Maskentragpflicht auf jene Mitarbeitenden zu beschränken, von denen tatsächlich ein Gefährdungspotential ausgeht, z. B. Mitarbeitende mit Patientenkontakt.

Nimmt man diese Interessenabwägung für die aktuelle Pandemische Grippe vor, so ist nach allem, was man bis heute weiss, doch von einem eher milden Verlauf auszugehen. Vor diesem Hintergrund scheint die Begründung einer generellen Impfpflicht allein gestützt auf das arbeitsrechtliche Weisungsrecht rechtlich kaum vertretbar. Hingegen ist die Anordnung einer Maskentragpflicht als mildere Massnahme schon jetzt als vom Weisungsrecht gedeckt anzusehen, jedenfalls für Mitarbeitende mit Patientenkontakt. Die Situation kann sich freilich rasch ändern, so z. B. wenn in einem Spital plötzlich mehrere akute Fälle auftreten und Gefahr besteht, dass die Verbreitung der Krankheit durch ungeimpfte Angestellte gefördert werden könnte. In solchen Konstellationen ist auch die Herleitung einer Impfpflicht via Weisungsrecht vorstellbar.

\section{Klarstellung}

Eine Klarstellung zum Schluss: Wenn in der öffentlichen Diskussion gelegentlich von einem «Impfzwang» die Rede ist, so ist gegen diesen Begriff dann nichts einzuwenden, wenn damit ein Zwang im Sinne einer rechtlichen Pflicht verstanden wird. Hingegen ist die Anwendung physischen Zwangs rechtlich so gut wie ausgeschlossen. Selbst das Epidemiegesetz kennt keine solchen Zwangsmassnahmen. Niemand muss also damit rechnen, gegen seinen Willen eine Impfung injiziert zu erhalten. Wenn sich ein Mitarbeiter weigert, sich einer rechtmässig angeordneten Impfung oder einer Maskentragpflicht zu unterziehen, riskiert er andere Rechtsnachteile, so z. B. die Einstellung der Lohnzahlung, wenn er wegen des von ihm ausgehenden Gefährdungspotentials nicht mehr am Arbeitsplatz eingesetzt werden kann. Allenfalls droht, wie im erwähnten St. Galler Fall, auch der Verlust der Stelle durch Kündigung.

\section{Literatur}

1 BGE 99 Ia $747 \mathrm{ff}$

2 St. Gallische Gerichts- und Verwaltungspraxis GVP 2006, Nr. 1. www.sg.ch/home/staat_recht/recht/GVP/ gvp_2006.Par.0001.DownloadListPar.0001.File.tmp/ GVP_2006_komplett.pdf 


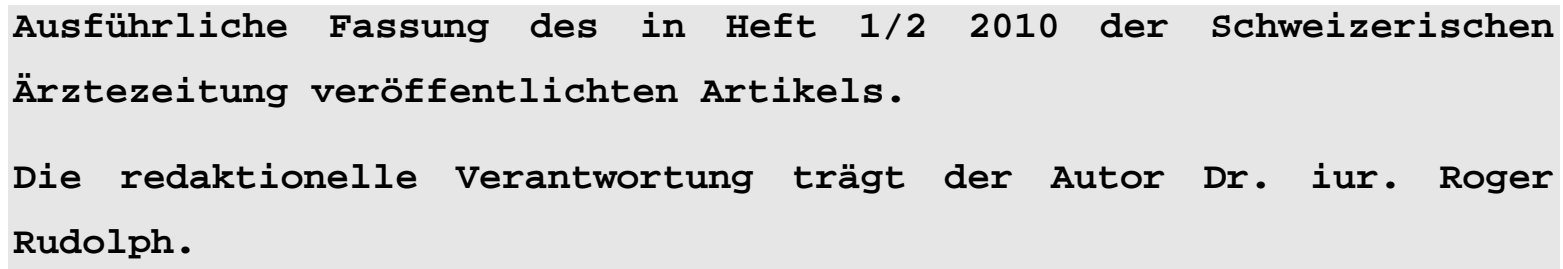

Pandemische Grippe (H1N1) 2009: Impfobligatorium für das Gesundheitspersonal?

\section{Ausgangslage}

Seit Anfang November 2009 hat die Anzahl bestätigter Fälle der pandemischen Grippe (H1N1) 2009, auch als Schweinegrippe bekannt, stark zugenommen ${ }^{1}$. Spätestens mit dem Bekanntwerden erster Todesfälle in Spitälern wurde der Ruf nach einem Impfobligatorium für die Beschäftigten im Gesundheitswesen laut. Damit einher ging eine intensiv und teils polemisch geführte Kontroverse zwischen Impfbefürwortern und Impfgegnern, die bis heute anhält. Während die Befürworter den Gegnern Unprofessionalität und Gleichgültigkeit gegenüber dem Patientenwohl vorwerfen, sehen die Gegner in der Forderung nach einem Impfobligatorium einen Angriff auf ihre körperliche Integrität und fundamentale verfassungsmässige Rechte. Zusätzlich angeheizt wurde die Diskussion, nachdem durch die Medien einem breiteren Publikum bekannt wurde, dass es um die Impfmoral der Pflegenden teils "katastrophal" bestellt sei². So wurde Ende November 2009 vermeldet, dass beispielsweise am Universitätsspital Zürich nur gerade 15\% des Pflegepersonals geimpft seien, während die Quote bei der Ärzteschaft immerhin rund 50\% erreiche.

Behörden, Politik und Verbände haben sich ebenfalls in die Diskussion eingeschaltet. Dabei ist bemerkenswert, dass selbst innerhalb der Bundesverwaltung unterschiedliche Standpunkte eingenommen werden. Während etwa das SECO die Möglichkeit eines

\footnotetext{
1 Dazu weiterführend der wöchentliche Situationsbericht des Bundesamtes für Gesundheit (BAG) : http://www.bag.admin.ch/influenza/06411/index.html?lang=de.

2 NZZ vom 28. November 2009, S. 17.
} 
Impfobligatoriums nach geltendem Recht pauschal ablehnt ${ }^{3}$ und auch die SUVA in ihren Empfehlungen zu Impfungen des Personals im Gesundheitsweisen ${ }^{4}$ die Durchsetzbarkeit einer Impfpflicht verneint, hat Bundesrat Burkhalter am 27. November 2009 eben dies gegenüber der "Tagesschau" des Schweizer Fernsehens gefordert, sollte sich die Bedrohungssituation verschärfen.

Während diese öffentliche Kontroverse weiterhin anhält, müssen die verantwortlichen Entscheidungsträger, also namentlich Spital- und Heimdirektionen sowie Inhaber von Arztpraxen, praxistaugliche Lösungen für den betrieblichen Alltag finden. Auch hier zeigt sich ein heterogenes Bild. Soweit ersichtlich, hat sich bis heute noch kein spital zu einem eigentlichen Impfobligatorium entschlossen. Anders sieht es bei der Maskentragpflicht als mildere Schutzmassnahme aus. Mitte November 2009 wurde bekannt, dass im Universitätsspital Genf die geimpften Mitarbeiter einen Ansteckpin tragen, der auf den Impfschutz hinweist, während das ungeimpfte Personal eine Gesichtsmaske tragen muss. Eine Differenzierung, welche dem Spital seitens der Gewerkschaften umgehend den Vorwurf der Diskriminierung und Erniedrigung eintrug. Eine Maskentragpflicht kennen unter anderem auch die spitäler des Kantons St. Gallen oder das Spital Uster. Andere Spitäler, wohl die Mehrheit, haben bis anhin von solchen Massnahmen abgesehen. Sie setzen stattdessen auf die Eigenverantwortung des Personals, wie z.B. das zu Hause bleiben bei Grippesymptomen, das freiwillige Maskentragen oder die strikte Einhaltung der Hygieneregeln.

\section{Unterschiedliche rechtliche Grundlagen}

Eine generelle Antwort auf die Frage, ob die aktuelle Situation ein Impfobligatorium rechtlich zulässt, fällt schwer. Das liegt schon daran, dass auf die Beschäftigungsverhältnisse im Gesundheitsbereich ganz unterschiedliche Rechtsnormen zur Anwendung kommen. Die Anstellungen der öffentlichrechtlichen Spitäler

\footnotetext{
3 FAQs "Pandemie und Betriebe"; http://www.seco.admin.ch/themen/00645/02337/index.html.

4 Broschüre "Impfungen des Personals im Gesundheitswesen", Bestellnummer: 2869/34.d, S. 11 .
} 
unterliegen in aller Regel kantonalen oder kommunalen Personalgesetzen bzw. -verordnungen, eigens für die betreffenden Spitäler geschaffenen öffentlichrechtlichen Personalrechten oder kollektivrechtlichen Vereinbarungen in Form Gesamtarbeitsverträgen. Die Anstellung selber erfolgt dabei entweder durch mitwirkungsbedürftige Verfügung oder durch verwaltungsrechtlichen Vertrag. Diese öffentlichrechtlichen Personalrechte sind vergleichsweise starr, indem sie individuellen Abweichungen vom übergeordneten Personalrecht nicht oder nur in engen Grenzen zugänglich sind. Dies ist eine direkte Folge des verfassungsmässigen Gleichbehandlungsgrundsatzes, welche jede Form des staatlichen Handelns prägt.

Demgegenüber unterstehen die Arbeitsverhältnisse der Privatspitäler sowie der Arztpraxen regelmässig dem privaten Arbeitsrecht des Obligationenrechts $(\mathrm{OR})^{5}$, wobei auch hier Gesamtarbeitsverträge möglich sind. Die Anstellung erfolgt durch einen privatrechtlichen Einzelarbeitsvertrag, in welchem häufig auf integrierende Vertragsbestandteile wie z.B. ein Mitarbeiterreglement verwiesen wird. Weil das OR vom Grundsatz der Vertragsfreiheit geprägt ist, können die Parteien auf diese Weise ihre gegenseitigen Rechte und Pflichten im Rahmen des zwingenden Gesetzesrechts frei und individuell gestalten. Ausserdem ist der privatrechtliche Gleichbehandlungsgrundsatz des OR ein vergleichsweise schwacher, so dass es ohne weiteres zulässig ist, selbst innerhalb ein- und derselben Mitarbeiterkategorie ganz unterschiedliche Vertragsabsprachen zu treffen.

\section{Impfobligatorium gestützt auf das Epidemiengesetz}

Generelle Aussagen sind aufgrund der Heterogenität der anwendbaren Rechtsgrundlagen also schwierig. Vergleichsweise klar ist die Rechtslage in Bezug auf das bundesrechtliche Epidemiengesetz, das

\footnotetext{
${ }^{5}$ Art. $319-362$ OR.
} 
derzeit zur Revision ansteht ${ }^{6}$. Der heute geltende Art. 23 räumt den Kantonen das Recht ein, ein Impfobligatorium gegen übertragbare Krankheiten auszusprechen, sofern für die Bevölkerung eine erhebliche Gefahr besteht ${ }^{7}$. Eine Rechtsgrundlage zur Einführung einer generellen Impfpflicht ist demnach, entgegen anderslautender Aussagen in der öffentlichen Diskussionen, bereits nach geltendem Recht durchaus gegeben. Allerdings ist es so, dass - soweit ersichtlich - in Bezug auf die aktuelle Grippepandemie noch von keinem Kanton ernsthaft erwogen worden ist, eine solche auf das Epidemiengesetz gestützte Impfpflicht tatsächlich auszusprechen. Die Kantone erachten die aktuelle Bedrohungssituation ganz offensichtlich als zu wenig bedrohlich, um einen so erheblichen Eingriff wie ein Impfobligatorium zu rechtfertigen.

\section{Weiterer Handlungsspielraum der Kantone}

Denkbar wäre auch, dass die Kantone ergänzend zum bundesrechtlichen Rahmen des Epidemiegesetzes Impfvorschriften für einzelne Bevölkerungsgruppen, also auch für das Gesundheitspersonal, erlassen, z.B. in den kantonalen Gesundheits- oder Personalgesetzen ${ }^{8}$. Davon haben die Kantone allerdings bis heute noch kaum Gebrauch gemacht, was sich im Zuge der aktuellen Pandemiediskussion allerdings ändern könnte. Eine solche Impfpflicht müsste, da sie die körperliche Integrität und damit das verfassungsmässige Grundrecht der persönlichen Freiheit tangiert ${ }^{9}$, gesetzlich abgestützt, von einem öffentlichen Interesse getragen und verhältnismässig $\operatorname{sein}^{10}$. Das Bundesgericht hat das Vorliegen dieser Voraussetzungen in einem älteren Entscheid aus dem Jahr 1979 ausdrücklich bejaht, als es ein generelles DiphtherieImpfobligatorium das Kantons waadt für Kinder für rechtmässig

6 Vgl. weiterführend zur Revision die Informationen auf der Website des BAG: http://www.bag.admin.ch/themen/medizin/00682/03877/05547/index.html?lang=de.

7 In ausserordentlichen Konstellationen kann auch der Bundesrat die nötigen Massnahmen anordnen (Art. 10 Epidemiegesetz).

8 Vorstellbar ist auch, dass mit eigener Satzungsautonomie ausgestattete öffentlichrechtliche Spitäler entsprechende Vorschriften in ihre eigenen Personalrechte aufnehmen.

9 Art. 10 Bundesverfassung.

10 Art. 36 Bundesverfassung. 
erklärte ${ }^{11}$. Das Bundesgericht erwog insbesondere, dass eine solche Impfung keine schwere Beeinträchtigung der körperlichen Integrität darstelle ("inoffensive et peu douloureuse"). Gleicher Meinung war das St. Galler Verwaltungsgerichts in einem Urteil vom 19. Oktober $2006^{12}$. Dieser Entscheid wurde in der öffentlichen Diskussion um ein mögliches Impfobligatorium für das Gesundheitspersonal bislang kaum beachtet, obwohl ihm genau ein solcher sachverhalt zugrunde lag. In diesem sorgfältig begründeten Urteil wurde die Weigerung einer Rotkreuzpflegehelferin am Kantonsspital St. Gallen, sich einer vorgeschriebenen Hepatitis B-Impfung zu unterziehen, als pflichtwidriges Verhalten taxiert. Die vom spital deswegen ausgesprochene Kündigung wurde folglich als gerechtfertigt beurteilt.

\section{Vertraglich vereinbarter Impfzwang?}

Eine interessante Frage ist, ob eine Impfpflicht auch durch vertragliche Vereinbarung zwischen Arbeitgeber und Arbeitnehmer verbindlich vereinbart werden kann. Eine solche Möglichkeit würde in erster Linie den privatrechtlichen Arbeitgebern zur Verfügung stehen, also den Privatspitälern und Arztpraxen. Denn das für sie massgebliche Obligationenrecht ist im Gegensatz zu den öffentlichrechtlichen Personalrechten vom Grundsatz der Vertragsfreiheit geprägt. Ausserdem sind privatrechtliche Arbeitgeber nicht im gleichen Masse den Grundrechten verpflichtet wie öffentlichrechtliche Institutionen ${ }^{13}$. Es stellt sich allerdings die Frage, ob eine solche vertragliche Impfverpflichtung mit dem Verbot übermässiger Selbstbindung von Art. 27 Abs. 2 des Zivilgesetzbuches (ZGB) vereinbar ist. Vereinfacht gesagt, schützt diese Bestimmung den Einzelnen vor sich selber, indem sie vertragliche Verpflichtungen, welche den Kernbereich der Persönlichkeit betreffen, unabhängig vom Willen der Vertragsparteien für unwirksam erklärt. Gestützt auf diese

\footnotetext{
11 BGE 99 Ia 747 ff.

12 St. Gallische Gerichts- und Verwaltungspraxis GVP 2006, Nr. 1. Abrufbar auf http://www.sg.ch/home/staat__recht/recht/GVP/gvp_2006.Par.0001.DownloadListPar.0001.File. tmp/GVP_2006_komplett.pdf.

13 Fehlen einer sogenannten direkten Drittwirkung im Privatbereich.
} 
Bestimmungen werden in der Literatur etwa vertragliche Verpflichtungen zur Einnahme von Verhütungsmitteln oder zum Blutspenden als unverbindlich erachtet, da sie einen Eingriff in die körperliche Integrität darstellten. Wie es sich in Bezug auf eine allfällige Impfpflicht für das Gesundheitspersonal verhält, muss einstweilen offenbleiben, da eine Rechtsprechung dazu ebenso wie eine wissenschaftliche Diskussion noch fehlt. Dessen ungeachtet könnte die vorsorgliche Aufnahme einer solchen Regelung in das Vertragswerk eine durchaus prüfenswerte Option darstellen.

Es zeigt sich also, dass Impfobligatorien gestützt auf das Epidemiegesetz oder anderweitige kantonalrechtliche Vorschriften bis heute entweder gar nicht erst erwogen werden oder aber dass die entsprechenden gesetzlichen Grundlagen erst noch geschaffen werden müssten. Und auch vertragliche Impfverpflichtungen sind, sofern man solche überhaupt für rechtlich zulässig erachtet, bislang kaum verbreitet. Damit verbleibt als letzter Anknüpfungspunkt für ein allfälliges Impfobligatorium nur noch das sogenannte Weisungsrecht des Arbeitgebers. Dies ist die Frage, auf die sich aus rechtlicher Sicht die aktuelle Diskussion um ein Impfobligatorium für das Gesundheitspersonal konzentrieren muss.

\section{Im Fokus: Das Weisungsrecht des Arbeitgebers}

Dieses Weisungsrecht ist für die privatrechtlichen Arbeitsverhältnisse in Art. 321d Abs. 1 OR ausdrücklich gesetzlich vorgesehen. Danach kann der Arbeitgeber über die Ausführung der Arbeit und das Verhalten des Arbeitnehmers am Arbeitsplatz allgemeine Anordnungen und individuelle Weisungen erlassen. Auch die öffentlichrechtlichen Personalrechte kennen vergleichbare Umschreibungen und Weisungsbefugnisse, teilweise verweisen sie auch einfach auf das OR. Unbestritten ist, dass auch der Schutz der Gesundheit von Arbeitnehmer, Mitarbeitenden und Kunden vom Weisungsbereich erfasst ist. Verschiedene Gesetzesbestimmungen verpflichten den Arbeitgeber sogar, Schutzvorkehrungen zu treffen, so z.B. die gesetzliche Fürsorgepflicht von Art. 328 OR, Art. 6 des Arbeitsgesetzes (ArG) oder Art. 82 Abs. 1 des 
Unfallversicherungsgesetzes (UVG). Man könnte sich fragen, ob sich gestützt auf diese Gesetzesbestimmungen sogar direkt eine Impfpflicht herleiten liesse ${ }^{14}$. Ein Gedanke, der allerdings bis heute in Literatur und Rechtsprechung noch kaum Niederschlag gefunden hat. Zumindest aber lässt sich sagen, dass das gesetzliche Weisungsrecht des Arbeitgebers als rechtsdogmatische Grundlage für ein Impfobligatorium herangezogen werden kann. Zum gleichen Schluss kam auch das st. Galler Verwaltungsgericht im bereits erwähnten Urteil. Die betroffene Mitarbeiterin hatte im Verfahren bestritten, dass sie der massgeblichen Weisung, welche die Impfverpflichtung enthielt, gültig zugestimmt habe, womit sie sinngemäss die Notwendigkeit einer vertraglichen Vereinbarung geltend machte. Damit drang sie nicht durch, denn das Gericht erwog, dass es darauf gar nicht ankomme. Es bejahte die Pflicht zur Vornahme der Hepatitis B-Impfung direkt gestützt auf das Weisungsrecht des Spitals und unabhängig davon, ob nun auch noch eine (vertragliche) Zustimmung vorlag oder nicht.

Wichtig ist nun aber, dass das Weisungsrecht des Arbeitgebers kein unbeschränktes ist. Es findet seine Grenzen insbesondere im Persönlichkeitsrecht des Arbeitnehmers (Art. 328 OR). Dabei ist immer eine Interessenabwägung vorzunehmen. Man kann in diesem Zusammenhang von einer dynamischen Begrenzung sprechen: Je intensiver das betriebliche Interesse, desto weiter darf in die Persönlichkeitsrechte eingegriffen werden ${ }^{15}$. Im Rahmen einer solchen Interessenabwägung kommt auf Arbeitnehmerseite dem Selbstbestimmungsrecht über die eigene körperliche Integrität überragende Bedeutung zu. Dieses wiegt zweifellos schwer, denn eine Spritzimpfung auf sich nehmen zu müssen, ist keine Bagatelle mehr, zumal auch Nebenwirkungen nicht ausgeschlossen werden können ${ }^{16}$. Aber auch aufseiten des Arbeitgebers fallen gewichtige Interessen an. In erster Linie ist sein legitimes Interesse an der

\footnotetext{
14 In diese Richtung geht eine der Argumentationsschienen im zuvor erwähnten St. Galler Entscheid.

15 Dazu Kommentar Streiff/von Kaenel, 6. Auflage, N 3 zu Art. 321d OR.

16 Gemäss einer Analyse von Swissmedic mussten bis Ende November 2009 allerdings kaum schwerere Komplikationen verzeichnet werden; dazu NZZ vom 5. Dezember 2009, S. 28.
} 
Aufrechterhaltung eines geordneten und sicheren Betriebs zu nennen. Dazu gehört auch der Schutz der Mitarbeitenden und Patienten vor vermeidbaren Gesundheitsgefährdungen. Was in diesem Zusammenhang oft vergessen wird ist, dass Arbeitgeber haftungs- und strafrechtliche Sanktionen riskieren, wenn sie es zulassen, dass in einer akuten Pandemiesituation infolge ungenügender Schutzvorkehrungen Patienten durch Ärzte, Praxisassistentinnen oder Pflegepersonal angesteckt werden und so zu Schaden kommen. Ein Risiko, das notabene auch den betroffenen Arbeitnehmern droht, etwa wenn ein ungeimpfter Arzt trotz offensichtlicher Krankheitssymptome zur Arbeit erscheint, Patienten betreut und diese dadurch ansteckt. Dieses latente Haftungsrisiko für Arbeitgeber wie Arbeitnehmer muss ebenfalls in die Interessenabwägung einfliessen.

Es liegt auf der Hand, dass die Gewichtung der einzelnen Faktoren massgeblich vom konkreten Bedrohungspotential einer Pandemie beeinflusst wird. Je mehr Fälle beispielsweise in einem spital oder einer Praxis auftreten, je schwerwiegender die symptome sind, je höher die Ansteckungsgefahr ist, je anfälliger die Patienten sind, um so eher wird das Arbeitgeberinteresse an einer Impfpflicht überwiegen, und vice versa. Weiter ist das Verhältnismässigkeitsgebot $\mathrm{zu}$ beachten. Daraus folgt, dass mildere Massnahmen, z.B. das Tragen von Masken, den Vorrang haben müssen, solange diese einen zuverlässigen Schutz gewährleisten können. Dies $\mathrm{zu}$ beurteilen, ist Sache der medizinischen Fachleute. Den behördlichen Empfehlungen kann dabei Richtliniencharakter zukommen. Sie entbinden die verantwortlichen Entscheidungsträger in einem Spital oder in einer Praxis aber nicht von einer selbständigen und laufenden Beurteilung der Bedrohungslage im eigenen Betrieb. Schliesslich haben sich einseitig vom Arbeitgeber angeordnete Massnahmen wie eine Impf- oder Maskentragpflicht auf jene Mitarbeitende zu beschränken, von denen tatsächlich ein Gefährdungspotential ausgeht. Bei Ärzten und Pflegenden mit direktem Patientenkontakt werden sich solche Massnahmen also eher rechtfertigen lassen als gegenüber dem kaufmännischen Personal im Innendienst. 
Wagt man nun den Versuch, diese Interessenabwägung für die aktuelle Pandemische Grippe (H1N1) 2009 vorzunehmen, so ist nach allem, was man bis heute über diese Grippe weiss, doch von einem eher milden Verlauf auszugehen. Vor diesem Hintergrund scheint die Begründung einer generellen Impfpflicht allein gestützt auf das arbeitsrechtliche Weisungsrecht aus heutiger sicht rechtlich kaum vertretbar. Hingegen ist die Anordnung einer Maskentragpflicht als mildere Massnahme schon jetzt als vom Weisungsrecht gedeckt anzusehen, jedenfalls für Mitarbeitende mit Patientenkontakt. Alternativ lässt sich eine solche Maskentragpflicht auch direkt aus den Bestimmungen des Arbeitsgesetzes und des Unfallversicherungsgesetzes sowie den dazugehörigen Verordnungen ableiten ${ }^{17}$. Die Situation kann sich freilich rasch ändern, so z.B. wenn in einem spital plötzlich mehrere akute Fälle auftreten und Gefahr besteht, dass die Verbreitung der Krankheit durch ungeimpfte Angestellte gefördert werden könnte. In solchen Konstellationen ist auch die Herleitung einer Impfpflicht via Weisungsrecht vorstellbar.

\section{Klarstellung}

Zum Schluss ist eine Klarstellung notwendig: Wenn in der öffentlichen Diskussion gelegentlich von einem "Impfzwang" die Rede ist, so ist gegen diesen Begriff dann nichts einzuwenden, wenn damit ein Zwang im Sinne einer rechtlichen Verpflichtung verstanden wird. Die vorstehenden Ausführungen zeigen, dass ein so verstandener Zwang unter bestimmten Voraussetzungen rechtlich begründbar ist. Hingegen ist ein Zwang in dem Sinn, dass Mitarbeiter physisch, notfalls unter Anwendung von behördlicher Gewalt, zu einer Impfung gezwungen werden, rechtlich so gut wie ausgeschlossen. Selbst das Epidemiegesetz kennt keinen solchen physischen Impfzwang und verzichtet auch darauf, die Verweigerung einer von den Kantonen angeordneten Zwangsimpfung mit Strafe zu bedrohen. Niemand muss also damit rechnen, gegen seinen Willen eine Impfung injiziert zu erhalten. Wenn sich ein Mitarbeiter weigert,

17 Art. 6 ArG in Verbindung mit Art. 10 und Art. 27 der Verordnung 3 zum ArG; Art. 82 Abs. 1 UVG in Verbindung mit Art. 11 Abs. 1 der Verordnung über die Unfallverhütung (VUV). 
sich einer rechtmässig angeordneten Impfung oder - als mildere Massnahme - einer Maskentragpflicht zu unterziehen, riskiert er andere Rechtsnachteile. Wenn eine Pflegeperson z.B. wegen des von ihr ausgehenden Gefährdungspotentials nicht mehr an ihrem Arbeitsplatz eingesetzt werden kann, muss sie damit rechnen, dass sie für diese Zeit wegen selbst verschuldeter Arbeitsverhinderung den Lohnanspruch verliert ${ }^{18}$. Allenfalls droht, wie im erwähnten st. Galler Fall, sogar eine Kündigung, die wegen Selbstverschulden nicht mehr erfolgreich angefochten werden könnte.

18 Die Situation ist hier vergleichbar mit dem Fall, dass ein erkrankter Arbeitnehmer eine ärztliche Behandlung oder die Einnahme von Medikamenten verweigert und dadurch die Arbeitsverhinderung verlängert wird. 